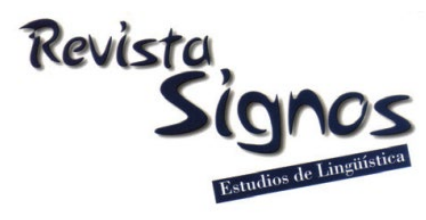

\title{
El uso de los tiempos de pasado en la construcción de la temporalidad en español L2 en niveles avanzados de competencia
}

\section{The use of Spanish past tenses in the construction of temporality in advanced learners of Spanish L.2}

\author{
Iban Mañas Navarrete Elisa Rosado Villegas Natalia Fullana Rivera \\ UNIVERSITAT DE BARCELONA \\ ESPAÑA \\ iban.manas@ub.edu \\ UNIVERSITAT DE BARCELONA \\ ESPAÑA \\ erosado@ub.edu \\ UNIVERSITAT DE BARCELONA \\ ESPAÑA \\ nataliafullana@ub.edu
}

Recibido: 29-V-2019 / Aceptado: 05-V-2020

DOI: $10.4067 /$ S0718-09342021000100054

\section{Resumen}

El objetivo de este trabajo es describir los rasgos que caracterizan el uso de los tiempos de pasado imperfecto e indefinido en español por parte de aprendices rusohablantes de nivel avanzado en situación de aprendizaje formal. Los datos analizados provienen de una tarea de producción escrita semiespontánea a partir de un estímulo audiovisual. El análisis de los resultados se aborda desde las categorías de la estructura narrativa y el aspecto léxico del verbo, con la intención de identificar su efecto en el uso de estos dos tiempos verbales. Los resultados revelan una preferencia unívoca por la actualización de los verbos a partir de combinaciones prototípicas entre aspecto gramatical, léxico y discursivo. Los niveles más altos de competencia muestran un dominio equivalente al nativo en el uso de imperfecto e indefinido a la hora de narrar eventos en pasado.

Palabras Clave: Español lengua extranjera, estructura temporal, aspecto léxico, pretérito imperfecto, indefinido. 


\begin{abstract}
The aim of the current research is to describe and analyze the specific characteristics of the use of the Spanish verbal past tenses, specifically the imperfecto and indefinido, by Russian advanced learners of Spanish L2 with high proficiency level in a formal learning environment. The collected data come from a semi-spontaneous written production task based on an audiovisual stimulus. The results are analyzed and categorized on the basis of the narrative structure and the lexical aspect of the verb, with the aim of identifying the effect of these factors on the use of past tenses verbs. The results reveal an unambiguous preference for the prototypical combinations between grammatical, lexical and discursive aspects across all groups. Moreover, the highly proficient L2 Spanish learners show a native-like performance with respect to the use of the Spanish imperfecto and indefinido in the narrative writing task.
\end{abstract}

Key Words: Spanish Foreign Language, temporality, lexical aspect, past tense, imperfecto-indefinido.

\title{
INTRODUCCIÓN
}

La interpretación y el uso de los sistemas de tiempo-aspecto-modo (TAM) en la adquisición de una segunda lengua (L2) es un fenómeno complejo que ha impulsado gran cantidad de trabajos, tanto de corte empírico como teórico, tal y como demuestra la publicación regular de compendios de estudios sobre esta cuestión (Salaberry \& Shirai, 2002; Labeau \& Saddour, 2012; Howard \& Leclercq, 2017). Este trabajo analiza la estructura narrativa para caracterizar el uso de dos tiempos de pasado del español, el pretérito imperfecto (IMP) y el indefinido (IND), en las producciones escritas de hablantes no nativos de niveles avanzados de competencia.

Este ha sido un tema recurrente en la investigación sobre adquisición del español como L2 (Montrul \& Salaberry, 2003; Baralo, 2007) principalmente en niveles iniciales e intermedios. Sin embargo, los trabajos centrados en niveles de competencia avanzados y muy avanzados son menos frecuentes (Montrul \& Slabakova, 2003; Salaberry, 2013), a pesar de que esta alternancia (IMP/IND) es uno de los fenómenos de la morfología funcional del verbo más difíciles de adquirir para los hablantes no nativos (Slabakova \& Montrul, 2008), y más problemáticas de tratar en el aula de 2L (Llopis-García, Real Espinosa \& Ruiz Campillo, 2012).

Este trabajo contribuye con un aporte empírico a esta línea de investigación con datos provenientes de hablantes rusófonos de español, perfil poco estudiado en el ámbito del español como L2 especialmente interesante debido a similitud parcial entre el sistema verbal ruso y español con respecto a los tiempos de pasado (Mañas, 2011).

\section{Marco teórico}

\subsection{La estructura narrativa en el uso de los tiempos de pasado}

Entre las hipótesis más destacadas sobre la adquisición de sistemas TAM encontramos la Hipótesis del Discurso (Discourse Hypothesis) (HD) (Bardovi-Harlig, 
1992, 1995). Esta hipótesis predice que, en los aprendices de una L2, la morfología verbal -que expresa el punto de vista aspectual, perfectivo o imperfectivo, de la situación (Smith, 1991) - emerge para distinguir entre primer plano, foreground, y segundo plano, background, de la estructura narrativa. Desde esta hipótesis, se considera el plano narrativo como una variable cuyo efecto en el uso de la morfología verbal se reduce conforme crece el dominio de la L2. Por tanto, para investigadores y profesores, lo relevante es dilucidar cómo esta distinción afecta a la selección y uso de la morfología verbal de pasado en hablantes no nativos.

Reinhart (1984), por su parte, sostiene que los rasgos que determinan la estructura principal se caracterizan a partir de: narratividad, puntualidad y completividad. Estas características coinciden con algunos rasgos que componen las diferentes clases del aspecto léxico verbal ( $\mathrm{Li} \&$ Shirai, 2000). Este hecho lleva a preguntarse sobre la relación entre la HD y los rasgos semánticos del verbo. Siguiendo la perspectiva tradicional (Comrie, 1976), los predicados se clasifican según su aspecto léxico en verbos télicos, que contienen un punto de culminación inherente -'sonreír,' 'despertar'-, y atélicos, que no marcan un límite inherente de la acción. Estos se suelen dividir en actividades, verbos dinámicos como 'pensar' o 'pasear', y no dinámicos, o estados, como 'ser' o 'tener'.

Si se acepta la existencia de esta conexión entre aspecto léxico y estructura narrativa, se asume una tendencia por parte de los predicados télicos a aparecer en el primer plano narrativo, mientras que la mayoría de verbos atélicos aparecerán en el segundo plano de la narración (Bardovi-Harlig, 2000). En su estudio sobre la relación entre la HD y el aspecto léxico en hablantes de neerlandés L2, Housen (1994) señala que la distinción foreground/background está marcada en la selección de verbos por el carácter aspectual inherente del predicado: los verbos puntuales y dinámicos aparecen con más frecuencia en el primer plano narrativo, y los de estado y durativos en el segundo plano. Resultados similares reporta López-Ortega (2000) sobre anglófonos aprendices de español L2.

En el caso de lenguas que expresan el aspecto gramatical de manera explícita, como el español con las formas de IMP e IND, el primer plano de la narración se construye principalmente con verbos télicos en forma perfectiva (IND), mientras el plano secundario combina verbos atélicos en forma imperfectiva, es decir, actualizados en IMP (Comajoan \& Pérez Saldanya, 2005). Bardovi-Harlig (1995) explica este fenómeno recurriendo a la funcionalidad del primer plano narrativo, especialmente transparente para los no nativos, pues hace avanzar la acción en un sentido lineal, concatenando verbos puntuales y télicos. Sin embargo, los eventos ubicados en el segundo plano no presentan necesariamente un orden canónico, por lo que los verbos de aspecto léxico carentes de límites permiten crear una línea temporal distinta a la principal. 


\subsection{La relación entre aspecto gramatical, aspecto léxico y discurso}

La HD experimenta un importante avance cuando considera la interacción entre los elementos lingüísticos que aportan información aspectual (Andersen \& Shirai, 1994). Las composiciones más frecuentes llevan a la conformación de combinaciones preferentes o prototípicas. A partir de estas observaciones, Shirai y Andersen (1995) formulan la Hipótesis del Prototipo (Prototype Hypothesis) (HP), que predice que "learners acquire a linguistic category starting with the prototype of the category and later expand its application to less prototypical cases" (Shirai \& Andersen, 1995: 758). Los casos prototípicos se encuentran en los verbos télicos ocupando la posición central de la narración actualizados con una forma perfectiva, mientras los estados y las actividades forman un marco de apoyo, y tienden a aparecer con formas imperfectivas (Andersen \& Shirai, 1994). Los ejemplos (1) y (2) ilustran una combinación prototípica y no prototípica respectivamente:

(1) La señora se tropezó y 'se cayó.'

(2) La señora, al ver que 'se caía', se agarró al brazo de su nieto.

En (1), 'caer' aparece actualizado en IND, de modo que la telicidad del aspecto léxico está en consonancia con la visión perfectiva de la acción que proyecta el IND. En cambio, en (2), la telicidad del verbo entra en conflicto con el IMP, que aplica una perspectiva imperfectiva, libre de un punto terminativo, de la acción. Así, la forma 'caía' presenta una situación de incongruencia o coacción aspectual (Swart, 1998) entre el aspecto léxico y el gramatical. Desde el punto de vista de la adquisición de L2, las combinaciones prototípicas son más transparentes para los aprendices que las incongruentes (Hasbún, 2000; Liskin-Gasparro, 2000), entre otras razones, por la baja frecuencia de estas combinaciones en la lengua (Ellis, 2013).

Salaberry y Comajoan (2013) optan por un enfoque multivariable por el alto potencial explicativo que éste ofrece. Igualmente, Comajoan y Pérez Saldanya (2005) y Salaberry (2011) advierten que una correcta interpretación de los datos debe considerar el nivel de la L2 de los hablantes, pues los efectos de las combinaciones prototípicas entre aspecto léxico y gramatical parecen tener más repercusión en estadios iniciales de adquisición, mientras que el impacto de la HD se registra en niveles posteriores de desarrollo (Bardovi-Harlig, 2000).

\section{Preguntas de investigación e hipótesis}

Tomando como punto de partida los presupuestos de la HD y de la HP, este trabajo se centra en determinar la influencia de la estructura narrativa y del aspecto léxico del verbo en la distribución de tiempos de pasado en los textos narrativos de estudiantes rusófonos de español L2 con un alto nivel de competencia. Asimismo, 
teniendo en cuenta los hallazgos de estudios anteriores sobre el tema (López Ortega, 2000; Amenós, 2010), planteamos la siguiente pregunta de investigación e hipótesis:

¿Qué papel juegan la distinción pragmático-discursiva entre primero y segundo plano informativo y el aspecto léxico del verbo en la distribución de tiempos de pasado en las narraciones de estudiantes rusófonos de español de niveles avanzados?

Hipótesis 1 (H1): En las producciones escritas de los hablantes no nativos, de todos los niveles de competencia, el indefinido suele aparecer en el primer plano narrativo y el imperfecto en el segundo plano.

Hipótesis 2 (H2): La aparición de predicados en pretérito imperfecto e indefinido en combinaciones no prototípicas entre aspecto léxico y discursivo aumenta conforme se incrementa el nivel de competencia de los aprendices.

\section{Metodología}

\subsection{Participantes}

La muestra del estudio está formada por 30 hablantes rusófonos de español como L2 (10 por grupo) y un grupo de control $(\mathrm{n}=10)$ de hablantes nativos $(\mathrm{HN})$ de español peninsular. Los hablantes no nativos son estudiantes de filología hispánica o traducción de la Universidad Estatal de San Petersburgo cuya primera lengua extranjera de trabajo es el español.

La distribución por niveles de competencia (B2, C1 y C2) se realizó a partir de la certificación de nivel de competencia de DELE y únicamente en el grupo de C2 se flexibilizó esta condición por la dificultad que supuso encontrar informantes con estas características. En este grupo se incluyeron tanto estudiantes de doctorado del Departamento de Traducción (español) como profesionales (traductores y profesores), con dominio probado del español.

\subsection{Instrumentos y procedimiento}

Se diseñó una tarea de producción escrita semicontrolada basada en un fragmento de la película muda Modern Times de Charles Chaplin, Alone and Hungry. El fragmento seleccionado ha sido empleado en estudios previos (Salaberry, 1999; Comajoan, 2001, citado en Comajoan \& Pérez Saldanya, 2005; Labeau, 2005) para estimular el uso de la expresión de pasado en diferentes lenguas.

La prueba se realizó a distancia a través de la plataforma virtual educativa Edmodo (Borg, O’Hara \& Hutter, 2008) en la que se creó un grupo de trabajo y se editó la tarea junto con todos los materiales necesarios para su elaboración. Tras el mensaje de bienvenida, se redactaron las instrucciones de la tarea, que incluyen un inicio de la narración ('un día una chica...'). Se decidió proponer este inicio para evitar el uso del presente histórico y guiar la producción de los informantes hacia el uso de formas de 
pasado (Salaberry, 1999). Para garantizar la similitud entre las producciones y, por tanto, una comparación más precisa entre las producciones, facilitamos a los informantes una serie de fotogramas del vídeo (Polio, 2012), 18 en total. La prueba no incluía límites de tiempo ni de extensión, únicamente se dio la instrucción explícita de que los textos incluyeran todas las escenas representadas en los fotogramas.

\subsection{Codificación y análisis}

Los textos obtenidos fueron transcritos, segmentados y codificados según las convenciones del formato CHAT de CHILDES (MacWhinney, 2000). No se realizó corrección ortográfica, aunque en los textos sí fueron normalizados errores léxicos para facilitar la comprensión. Se codificaron únicamente las formas verbales conjugadas en imperfecto e indefinido y en cada forma se clasificó el plano discursivo en el que se encontraba: primer plano (Foreground $(\mathrm{F})$ ) y segundo plano (Background (B)). A continuación, se recogió el tiempo verbal en el que estaban actualizadas: Indefinido $(\mathrm{N})$ o Imperfecto $(\mathrm{M})$; por último, se marcó la clase de aspecto léxico de cada verbo: atélicos (estados (s) y actividades (a)), y télicos (eventos (e)).

Los datos fueron codificados primeramente por un único investigador. Posteriormente se seleccionó un 30\% del corpus para que fuera codificado por otros dos investigadores. El nivel de coincidencia entre las codificaciones de los tres evaluadores se calculó con la prueba de Kappa de Fleiss $(K=0,837, p=0,000)$ que mostró un índice alto de coincidencia entre codificadores. Todos los contextos dudosos se discutieron y consensuaron entre los tres evaluadores.

Para el análisis del total de verbos en IMP e IND realizamos un ANOVA unifactorial con el nivel de competencia como factor para las cuatro condiciones resultado de la combinación de la forma verbal y el plano narrativo (FN, FM, BN y BM) en SPSS v.21 para Windows. Posteriormente, se realizó un ANOVA de medidas repetidas para determinar el efecto de las dos variables independientes: estructura narrativa (F, B) y aspecto léxico (s, a, e), en el uso de los tiempos de pasado. Se investigó, asimismo, la existencia de una posible interacción entre el aspecto léxico de un verbo y la estructura narrativa, y su influencia en la selección de IMP e IND.

\section{Resultados}

Para establecer una visión general del uso de los dos tiempos de pasado, partimos del análisis del total de formas verbales en IMP e IND. Dada la diferente extensión de los textos, se muestra la distribución de los tiempos verbales en porcentajes, así como los estadísticos descriptivos (media y desviación típica) correspondientes. 
Tabla 1. Porcentajes y estadísticos descriptivos (media y DT) de verbos en IMP e IND según estructura narrativa.

\begin{tabular}{|c|c|c|c|c|c|c|c|c|}
\hline & \multicolumn{2}{|c|}{ FN } & \multicolumn{2}{|c|}{ BN } & \multicolumn{2}{|c|}{ FM } & \multicolumn{2}{|c|}{ BM } \\
\hline & $\%$ & $\begin{array}{c}M \\
(D T)\end{array}$ & $\%$ & $\begin{array}{c}M \\
(D T)\end{array}$ & $\%$ & $\begin{array}{c}M \\
(D T)\end{array}$ & $\%$ & $\begin{array}{c}M \\
(D T)\end{array}$ \\
\hline \multirow{2}{*}{ HN } & \multirow{2}{*}{66,9} & 66,4 & \multirow{2}{*}{8,2} & 7 & \multirow{2}{*}{7,9} & 9,3 & \multirow{2}{*}{15,8} & 17 \\
\hline & & $(23.47)$ & & $-6,09$ & & $-20,35$ & & $-6,17$ \\
\hline \multirow{2}{*}{$\mathrm{C} 2$} & \multirow{2}{*}{73.3} & 73,7 & \multirow{2}{*}{6,8} & 7,5 & \multirow{2}{*}{6,2} & 6 & \multirow{2}{*}{13,7} & 13,4 \\
\hline & & $-10,54$ & & $-3,9$ & & $-3,08$ & & $-7,15$ \\
\hline \multirow{2}{*}{ C1 } & \multirow{2}{*}{71.6} & 72,3 & \multirow{2}{*}{3,7} & 3,9 & \multirow{2}{*}{8,7} & 9,3 & \multirow{2}{*}{15,9} & 15,7 \\
\hline & & $-11,27$ & & $-3,66$ & & $-9,63$ & & $-5,61$ \\
\hline \multirow{2}{*}{ B2 } & \multirow{2}{*}{71,6} & 72,4 & \multirow{2}{*}{2,9} & 3,2 & \multirow{2}{*}{11,3} & 11,9 & \multirow{2}{*}{14,1} & 15,2 \\
\hline & & $-9,99$ & & $-2,93$ & & $-10,49$ & & $-6,12$ \\
\hline
\end{tabular}

Nota: HN: grupo control; C2: hablantes rusófonos nivel C2; C1: hablantes rusófonos nivel C1; hablantes rusófonos nivel B2; FN: indefinido en foreground; BN: indefinido en background; FM: imperfecto en foreground; BM: imperfecto en background.

Los resultados de la Tabla 1 muestran la preeminencia del IND en el primer plano discursivo $(\mathrm{FN})$ en todos los grupos y en un porcentaje muy similar entre ellos: entre los grupos de no nativos supera ligeramente el 70\% y en el grupo $\mathrm{HN}$ se registra un índice algo inferior $(M=66,4)$. Teniendo en cuenta que el IND es el tiempo que crea el esqueleto de la trama (Ortiz Gonzalo, 1994), resulta esperable, como formulamos en la H1, que al narrar una historia los participantes se apoyen principalmente en este tiempo verbal y esta función discursiva. El IMP ocupa una posición mucho más discreta en la configuración de este plano narrativo (FM). Entre los grupos no nativos se observa un decrecimiento del uso del imperfecto conforme aumenta el nivel de L2. La creciente complejidad sintáctica de las producciones, que enriquece el plano secundario de la narración mediante la profusión de construcciones subordinadas, podría explicar esta tendencia.

En cuanto al plano de fondo, se observa un mayor uso del IMP en todos los grupos, reflejando el índice más alto el grupo HN. Las medias de los grupos $\mathrm{HN}(M=$ 17) y $\mathrm{C} 2(M=13,4)$ en IMP representan cerca del doble de las formas del IND en el mismo plano $(M=7$, para $\mathrm{HN} ; M=7,5$, para $\mathrm{C} 2)$. En los grupos B2 y C1, el uso del IND es prácticamente anecdótico: un $2,9 \%$ y un 3,7\%, respectivamente. Estos resultados se ajustan a la $\mathrm{HD}$, que predice que los tiempos verbales perfectivos aparecerán principalmente en el primer plano del discurso, mientras que los imperfectivos serán característicos del segundo plano.

Respecto a la combinación entre los dos planos de la narración ( $\mathrm{F}$ y B), el grupo B2 presenta el desequilibrio más acusado: la mayoría de formas verbales en indefinido e imperfecto ( $\mathrm{N}$ y $\mathrm{M}$ ) conforma el primer plano narrativo (F) (82,9\%) en detrimento del plano secundario, cuya configuración cuenta con el porcentaje de pasados más bajo (17,1\%). A su vez, los textos de los nativos parecen los más equilibrados en cuanto a la distribución entre el plano de foco narrativo (B) $(74,8 \%)$ y el plano de fondo (B) (24\%). 
Para poder confirmar si estas diferencias entre grupos para las cuatro condiciones eran significativas, comprobamos si la distribución de los datos se ajustaba a una distribución normal aplicando el test Shapiro-Wilk. Los resultados muestran una distribución normal para el plano secundario, tanto en IMP como en IND. En el primer plano de la narración los datos del grupo nativo no siguen una distribución normal ni para IND $(W=0,736, p=0,002)$ ni para IMP $(W=0,494 p=0,000)$. Para esta última condición, el grupo C1 tampoco presenta una distribución normal $(W=$ $0,735, p=0,002)$.

Estos resultados muestran la preeminencia del IND para el primer plano narrativo, en detrimento de la conjugación de verbos en IMP. Seguidamente se realizó un ANOVA de un factor para comparar el uso de estos dos tiempos verbales entre los grupos en las cuatro condiciones. El análisis no reveló diferencias significativas entre los grupos para ninguna de las condiciones: $\mathrm{FN}\left(F(3,36)=0,376, p=0,771, \eta^{2}=\right.$ $0,03), \operatorname{FM}\left(F(3,36)=0,361, p=0,782, \eta^{2}=0,02\right), \mathrm{BN}\left(F(3,36)=2,531, p=0,072, \eta^{2}=\right.$ $0,17), \mathrm{BM}\left(F(3,36)=0,563, p=0,643, \eta^{2}=0,04\right)$. Con estos resultados, podemos afirmar que, en las producciones de nuestro corpus, el indefinido es la forma más utilizada por todos los grupos, superando porcentualmente el número de formas en imperfecto. Se confirma asimismo que las producciones de los cuatro grupos presentan unos porcentajes similares (ver Tabla 1) entre formas verbales y planos narrativos.

Posteriormente, llevamos a cabo un nuevo recuento del total de verbos que aparecen en IMP e IND que fueron categorizados según aspecto léxico y plano narrativo. La tabla 2 resume esta recategorización expresada en total de observaciones y porcentajes. Los estadísticos descriptivos (media y desviación típica) de la Tabla 3 se calcularon a partir de los porcentajes y no del total de observaciones de la Tabla 2.

Tabla 2. Total de observaciones y porcentajes (en paréntesis) de verbos en IMP e IND según aspecto léxico.

\begin{tabular}{|l|c|c|c|c|c|c|}
\cline { 2 - 7 } \multicolumn{1}{c|}{} & \multicolumn{3}{c|}{ IND } & \multicolumn{3}{c|}{ IMP } \\
\hline \multirow{3}{*}{$\mathbf{H N}$} & $\mathbf{e}$ & $\mathbf{a}$ & $\mathbf{s}$ & $\mathbf{e}$ & $\mathbf{a}$ & $\mathbf{s}$ \\
\cline { 2 - 7 } & 155 & 30 & 24 & 26 & 15 & 25 \\
\hline \multirow{2}{*}{$\mathbf{C 2}$} & $-56,3$ & $-10,8$ & $-8,7$ & $-9,4$ & $-5,5$ & $-9,1$ \\
\hline \multirow{2}{*}{$\mathbf{C 1}$} & 277 & 54 & 18 & 7 & 20 & 59 \\
\hline \multirow{2}{*}{ B2 } & $-63,7$ & $-12,4$ & $-4,2$ & $-1,5$ & $-4,6$ & $(13.5)$ \\
\cline { 2 - 7 } & 327 & 35 & 21 & 15 & 24 & 77 \\
\cline { 2 - 7 } & $-65,6$ & -7 & $-4,2$ & -3 & $-4,8$ & $-15,4$ \\
\cline { 2 - 7 } & 300 & 15 & 9 & 4 & 20 & 87 \\
\hline
\end{tabular}

Nota: HN: grupo control; C2: hablantes rusófonos nivel C2; C1: hablantes rusófonos nivel C1; hablantes rusófonos nivel B2; FN: indefinido en foreground; BN: indefinido en background; FM: imperfecto en foreground; BM: imperfecto en background. 
En la Tabla 2 se percibe como el número de eventos en IND tiende a disminuir conforme avanza el nivel de competencia. Mientras se reduce la cantidad de formas verbales en IND con verbos télicos, aumentan las combinaciones aspectuales menos prototípicas. La actualización de verbos de estado en IND es prácticamente inexistente en el grupo B2 con únicamente el 2\% de la producción de verbos en este tiempo. Este porcentaje aumenta hasta alcanzar un $8,7 \%$ dentro del grupo $\mathrm{HN}$. El grupo B2 muestran una preferencia casi exclusiva por los verbos télicos al construir predicados en IND.

Entre los grupos no nativos, el segundo tipo de predicado más frecuente es la otra combinación prototípica entre aspecto gramatical y aspecto léxico: verbos de estado en IMP. Estos dominan el total de verbos en IMP con amplia ventaja sobre el resto de tipos de aspecto léxico. Por su parte, la combinación con una frecuencia más baja, no solo de los predicados en IMP, sino de todo el conjunto de verbos, es la que representa verbos télicos en IMP. La incidencia de esta combinatoria es anecdótica entre los no nativos, alcanzando su porcentaje más alto en el grupo C1 (3\%) y apareciendo testimonialmente en B2 (0,9\%).

Observamos que la distribución de los verbos de estado en IND e IMP presenta un comportamiento similar entre los grupos no nativos: la combinación verbos de estado en IMP resulta mucho más frecuente que en IND. Para los estudiantes de B2, los estados en IND suponen únicamente el $2 \%$, mientras que en IMP los verbos estativos llegan a un 18,1\%. Los grupos C1 y C2 registran un mismo porcentaje $(4,2 \%)$ de estados en IND, resultado muy inferior a su equivalente en IMP, que en C1 se sitúa en el 15,4\% y en C2 supone el 13,5\% del total de verbos. Podemos notar cómo, de manera gradual, la aparición de combinaciones aspectuales no prototípicas se incrementa con el nivel de LE.

Las actividades muestran un patrón singular de distribución de las dos formas verbales. Estas, al igual que los estados, están constituidas por verbos atélicos, lo que favorece su aparición en imperfecto; si bien la relación entre aspecto léxico y aspecto gramatical en las actividades es probablemente más débil que la de los estados, debido principalmente a la influencia de los complementos verbales sobre la estructura aspectual de las actividades, convirtiéndolas en eventos, en verbos télicos (Díaz, Bel, Ruggia, Bekiou \& Rosado, 2003). En nuestro corpus, en cambio, las actividades son empleadas en IND en un porcentaje más alto que en IMP. Esta tendencia aparece de manera transversal en todos los grupos, excepto en B2, en cuyas producciones se contabilizó un $3,5 \%$ de actividades en IND y un $4,7 \%$ en IMP. Se puede presumir, pues, que para los hablantes no nativos por encima de B2, el aspecto léxico de las actividades no guía la selección del tiempo verbal en esta clase aspectual.

Para comprobar la incidencia de la estructura del discurso en la selección del tiempo verbal, realizamos un ANOVA de medidas repetidas con dos variables 
dependientes: el tiempo verbal seleccionado (IND e IMP) y el aspecto léxico del verbo (e, a y s), con el nivel de competencia y el plano de la estructura narrativa como factores inter-sujetos. La tabla 3 muestra los estadísticos descriptivos (media y desviación típica).

Tabla 3. Distribuciones medias de las formas verbales (DT entre paréntesis) según aspecto léxico y estructura narrativa para todos los grupos.

\begin{tabular}{|c|c|c|c|c|c|c|c|c|c|c|c|c|}
\hline & \multicolumn{2}{|c|}{ IND_e } & \multicolumn{2}{|c|}{ IND_a } & \multicolumn{2}{|c|}{ IND_s } & \multicolumn{2}{|c|}{ IMP_e } & \multicolumn{2}{|c|}{ IMP_a } & \multicolumn{2}{|c|}{ IMP_s } \\
\hline & $\mathbf{F}$ & B & $\mathbf{F}$ & B & F & B & $\mathbf{F}$ & B & F & B & F & B \\
\hline \multirow{2}{*}{$\mathbf{H N}$} & 52,4 & 3,39 & 8,83 & 1,45 & 5,17 & 2,21 & 6,52 & 4,9 & 0,71 & 5,57 & 2,07 & 6,55 \\
\hline & $-18,43$ & $-3,37$ & $-4,66$ & $-1,73$ & $-4,45$ & $-2,99$ & $-19,19$ & $-2,49$ & $-1,17$ & $-3,41$ & $-2,71$ & $-4,7$ \\
\hline \multirow{2}{*}{ C2 } & 58,88 & 5,71 & 11,43 & 0,86 & 3,43 & 0,5 & 0,39 & 0,86 & 2,05 & 2,76 & 3,62 & 9,57 \\
\hline & $-11,32$ & $-3,42$ & $-5,6$ & $-1,25$ & $-1,92$ & $-1,11$ & $-0,87$ & $-1,39$ & $-1,53$ & $-2,7$ & $-3,28$ & $-7,09$ \\
\hline \multirow{2}{*}{ C1 } & 62,31 & 2,62 & 6,06 & 0,85 & 3,93 & 0,43 & 0 & 3,06 & 1,68 & 3,02 & 7,5 & 8,46 \\
\hline & $-9,55$ & $-2,63$ & $-3,87$ & $-1,1$ & $-1,98$ & $-1,35$ & 0 & $-1,75$ & $-1,57$ & $-1,68$ & $-9,2$ & $-5,26$ \\
\hline \multirow{2}{*}{ B2 } & 68,06 & 2,23 & 2,65 & 0,43 & 1,72 & 0,57 & 0,38 & 0,67 & 3,2 & 2,52 & 8,28 & 9,19 \\
\hline & $-13,04$ & $-2,35$ & $-3,73$ & $-0,91$ & -3 & $-1,22$ & $-1,2$ & $-1,56$ & $-4,87$ & $-3,12$ & $-9,79$ & $-5,09$ \\
\hline
\end{tabular}

Nota: HN: grupo control; C2: hablantes rusófonos nivel C2; C1: hablantes rusófonos nivel C1; hablantes rusófonos nivel B2; FN: indefinido en foreground; BN: indefinido en background; FM: imperfecto en foreground; BM: imperfecto en background.

Las pruebas de efectos intrasujetos se midieron a partir de la corrección Greenhouse-Geisser, puesto que se rechazó la hipótesis de esfericidad tanto para la variable aspecto léxico $\left(\chi^{2}(2)=10,27, p=0,006\right)$ como para la interacción entre esta variable y la selección del tiempo verbal $\left(\chi^{2}(2)=40,18, p=0,000\right)$. El ANOVA de medidas repetidas señaló una interacción significativa, con un tamaño del efecto bajo, entre las cuatro variables del estudio $\left(F(4.19,100.55)=3,06, p=0,018, \eta^{2}=0,11\right.$, potencia $=0,80)$; así, entre los cuatro grupos existen diferencias significativas en la selección de formas de IND e IMP. Actúan también de manera distinta con respecto a la distribución de estas formas en la construcción narrativa, entre primer y segundo plano del discurso, y en relación con el aspecto léxico.

El ANOVA de medidas repetidas también mostró una interacción significativa entre aspecto léxico y nivel de competencia en la selección de la forma verbal $(F(4.19$, $100.55)=4,62, p=0,002, \eta^{2}=0,16$, potencia $\left.=0,94\right)$. Por su parte, el efecto de la estructura narrativa fue analizado a través de una prueba de efectos intersujeto, cuyos resultados señalaron un efecto significativo de esta interacción $(F(3,72)=4,58, p=$ $0,005, \eta^{2}=0,16$, potencia $\left.=0,87\right)$. Es decir, tanto el aspecto léxico como la estructura narrativa determinan significativamente el empleo que hacen de IMP e IND los participantes del estudio, lo cual se ajusta a la predicción de la H2. Los Gráficos 1 y 2 muestran la interacción entre aspecto léxico y nivel de competencia en la selección de IND e IMP. 


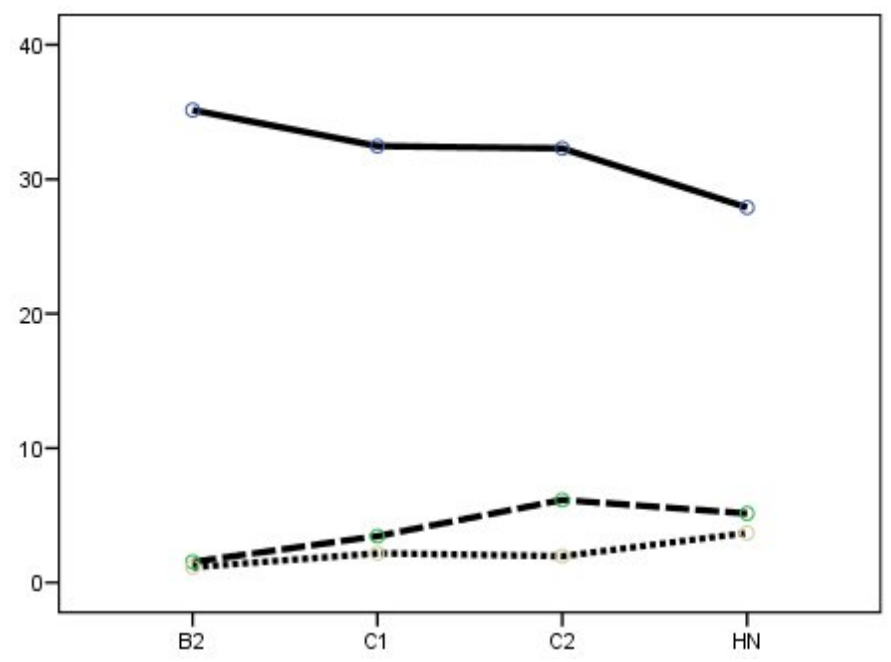

Gráfico 1. Interacción del aspecto léxico y el nivel de L2 para la selección de verbos en IND. Nota: HN: grupo control; C2: hablantes rusófonos nivel C2; C1: hablantes rusófonos nivel $\mathrm{C} 1$; hablantes rusófonos nivel B2.

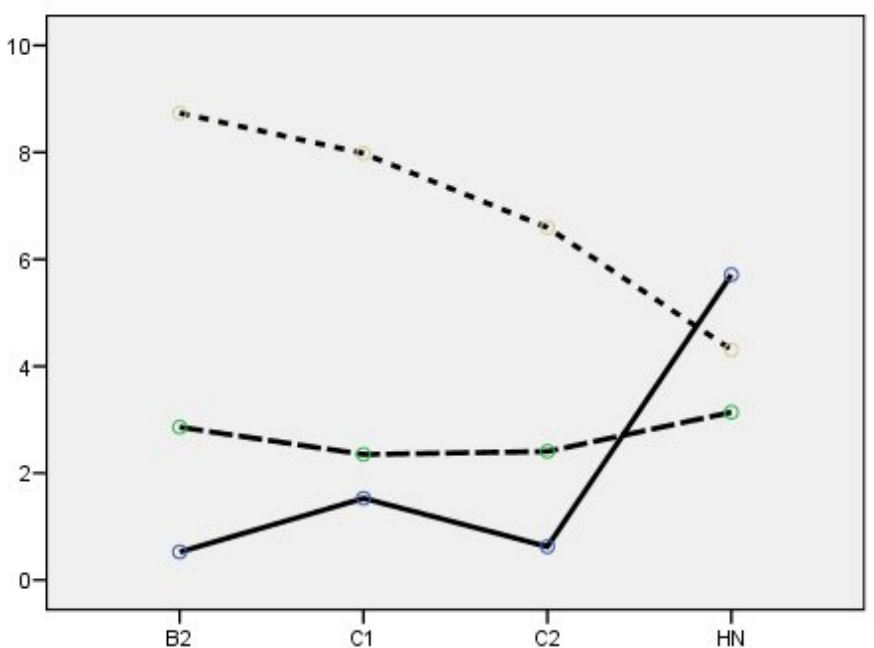

aspecto léxico

- eventos - actividades - - estados

Gráfico 2. Interacción del aspecto léxico y el nivel de L2 para la selección de verbos en IMP. Nota: HN: grupo control; C2: hablantes rusófonos nivel C2; C1: hablantes rusófonos nivel $\mathrm{C} 1$; hablantes rusófonos nivel $\mathrm{B} 2$.

En el Gráfico 1 se observa una disminución progresiva de los verbos de aspecto télico en IND. Los estados y las actividades tienen una presencia más baja, aunque estas últimas registran un incremento destacable en los grupos C2 $(6,1)$ y $\mathrm{HN}(5,1)$. Es en el grupo C2 donde se registran más predicados no prototípicos, como el de (3): 
(3) *RMB: La pareja se sentó (FNe) a descansar al lado de una casa y contemplaron $(\mathrm{FNa})$ a una señora en el umbral despedirse de su marido.

El número de verbos de estado en IMP (Gráfico 2) se reduce también conforme aumenta el nivel de competencia. Los eventos en IMP, resultado del aumento acusado que experimentan en el grupo $\mathrm{HN}(5,7)$, superan a los tipos de verbos atélicos $(4,3)$, dando lugar a una interacción no ordinal entre aspecto léxico y nivel de competencia. $\mathrm{El}$ incremento de eventos en IMP dentro del grupo $\mathrm{HN}$ se debe principalmente al uso del imperfecto narrativo en algunas producciones.

Las comparaciones por pares con el ajuste de Bonferroni señalaron diferencias significativas únicamente en las actividades entre los grupos B2 y C1 $(p=0,004), \mathrm{y}$ entre $\mathrm{B} 2$ y HN $(p=0,008)$. Con respecto a la interacción entre aspecto léxico y formas verbales, todos los contrastes, corregidos con Bonferroni, fueron significativos: para los estados $(p=0,000)$, las actividades $(\phi=0,004)$ y los eventos $(\phi=0,000)$. Es decir, las tres clases aspectuales presentan un comportamiento estadísticamente diferente según aparezcan en IMP o IND en todos los grupos.

En cuanto a la estructura narrativa, el análisis refleja la distribución del IND y el IMP para la construcción del primer y el segundo plano narrativo, y cómo evoluciona esa distribución entre los diferentes niveles de competencia. Los Gráficos 3 y 4 muestran la selección del IND y el IMP para todos los grupos.

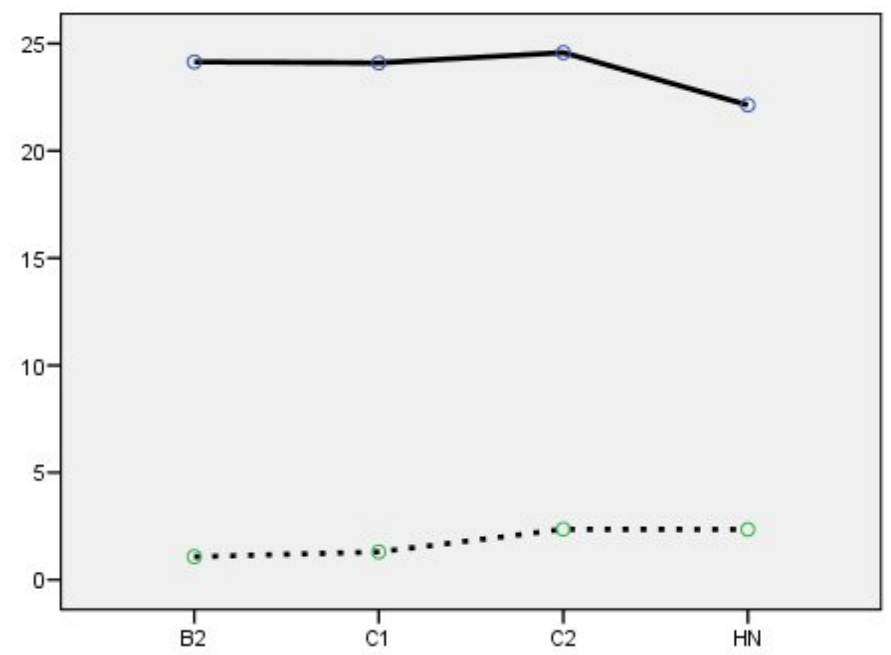

plano de discurso

- foreground

... background

Gráfico 3. Interacción de la estructura narrativa y el nivel de L2 para la selección de verbos en IND.

Nota: HN: grupo control; C2: hablantes rusófonos nivel C2; C1: hablantes rusófonos nivel

C1; hablantes rusófonos nivel B2. 


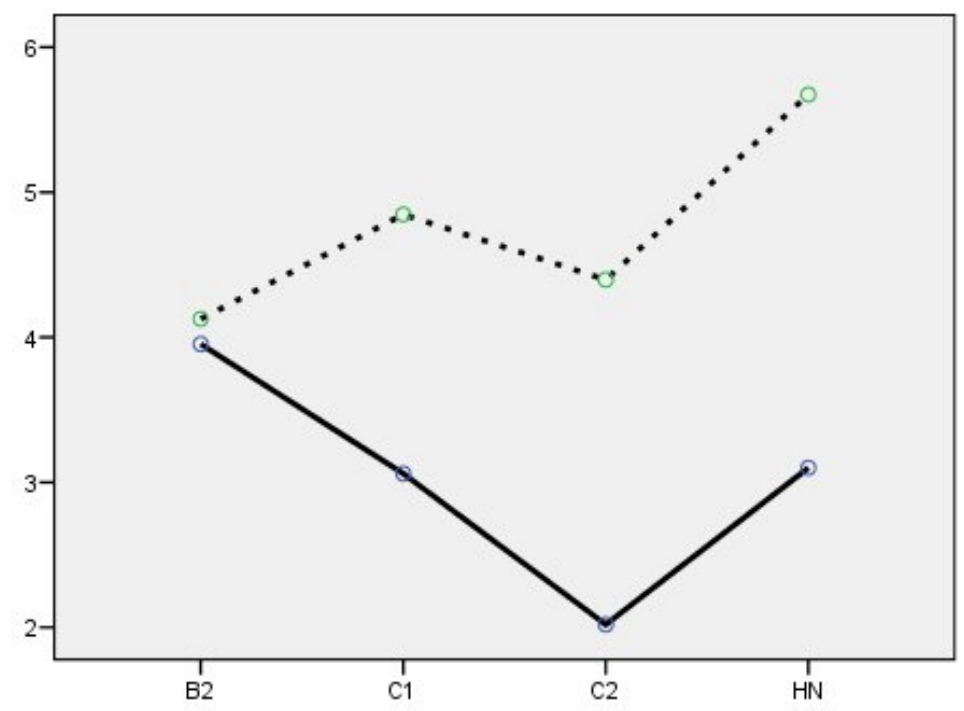

Gráfico 4. Interacción de la estructura narrativa y el nivel de L2 para la selección de verbos en IMP.

Nota: HN: grupo control; C2: hablantes rusófonos nivel C2; C1: hablantes rusófonos nivel $\mathrm{C} 1$; hablantes rusófonos nivel $\mathrm{B} 2$.

Entre los verbos en IND destaca la estabilidad que presentan los resultados en la creación del primer y el segundo plano narrativo en todos los grupos (Gráfico 3). Se observa, en la línea del primer plano de la narración (F), un ligero descenso del IND por parte del grupo HN (22,13). En cambio, en el empleo del IMP (Gráfico 4) se observa más claramente una interacción entre plano narrativo y nivel de competencia. Así, en el grupo B2, la presencia del IMP en los dos planos del discurso exhibe unos valores muy cercanos $(\mathrm{IMP}=4,13 ; \mathrm{IND}=3,95)$. Entendemos que estos datos pueden reflejar la complejidad sintáctica de las producciones escritas, en concreto, de la ausencia de subordinación, localización más habitual del IMP en la narración (Salaberry, 1999). El ejemplo de (4), de B2, ilustra esta observación:

(4) *RAH: Por supuesto, el policía tenía que llevarlo (FMs) a la presion [: prisión].

*RAH: En el coche de policía Charlie Chaplin se encontró (FNe) con esta pobre joven, le cedió (FNe) el sitio, le prestaba (FMa) la atención, le miraba (FMa) atentamente y con amabilidad.

Como vemos, la concatenación de frases yuxtapuestas, que bien podría deberse a un efecto del diseño de la tarea, no permite establecer una diferencia clara entre planos discursivos, entre información principal y complementaria. Se obtiene así una estructura lineal del texto en la que las acciones se suceden, una tras otra, en un único hilo narrativo, sin un contexto de fondo que permita alternar los dos planos del discurso. Por su parte, en los grupos $\mathrm{C} 1$ y C2 se advierte una preferencia por el IMP 
para la elaboración del plano secundario. Este comportamiento los acerca a la actuación de los nativos y marca un desarrollo que evoluciona de manera análoga entre estos tres grupos.

Las comparaciones por pares con el ajuste de Bonferroni identificaron diferencias significativas en el empleo de IND e IMP entre el grupo B2 y los nativos en la construcción del primer plano narrativo $(\mathrm{F})(p=0,012)$, así como en la construcción del segundo plano (B) $(p=0,013)$. No se registraron diferencias significativas entre los grupos de $\mathrm{C} 1, \mathrm{C} 2$ y $\mathrm{HN}$, en línea con lo mencionado anteriormente acerca del comportamiento similar de los grupos de más avanzados y los nativos en el uso de tiempos verbales y la construcción de planos narrativos.

\section{Discusión}

El análisis de los resultados aquí expuesto permite definir el papel de la estructura narrativa y el aspecto léxico en la selección verbal entre IMP e IND en niveles avanzados en español L2. Los resultados muestran que el empleo de estos dos tiempos verbales sigue un patrón muy similar en las narraciones de los cuatro grupos. Destaca una prevalencia del IND en función narrativa entre todos los grupos. También se detecta una tendencia a construir el segundo plano con formas de IMP, siendo anecdótica la presencia del IND, especialmente en los grupos B2 y C1. Se produce, así, un incremento del número de verbos que conforman el plano de fondo según aumenta el nivel de competencia en L2. Estos resultados coinciden con los de estudios previos (Liskin-Gasparro, 1996; Güell, 1998) y confirman los supuestos de la HD, nuestra primera hipótesis. Asimismo, se observa una cierta evolución de los grupos no nativos, cuyas distribuciones se acercan gradualmente a la de los nativos, en la línea de Schell (2000) y Cadierno (2000).

Si bien el análisis de la distribución de formas en IMP e IND entre los dos planos narrativos no señaló diferencias significativas entre los grupos, sí aparecieron en el ANOVA de medidas repetidas en el uso de las formas verbales para la composición de la estructura narrativa entre el grupo B2 y los nativos. Podemos señalar, pues, que, a pesar de la preeminencia del IND en el primer plano discursivo y del IMP en el segundo, la construcción de la estructura narrativa difiere entre los hablantes de B2 y el resto de grupos. Los grupos de nivel C1 y C2 muestran una competencia equivalente a la de los nativos.

Al tomar en consideración las variables aspecto léxico y estructura narrativa, se encontró una interacción entre el aspecto léxico y la estructura narrativa en la distribución del IMP y el IND en los diferentes niveles de competencia, así como una diferencia significativa de la distribución del IMP y el IND en todas las clases de aspecto léxico. Estos resultados siguen las predicciones marcadas por la HP. Sobre esta misma idea, en Hasbún (2000: 236) se concluye que "si en el habla de los nativos ocurre un sesgo [...], entonces los aprendices son inducidos a hacer relaciones 
inapropiadas entre forma y significado". Nuestros resultados están en esta línea, en tanto que el patrón que establecen los nativos en sus narraciones entre aspecto léxico, plano narrativo y selección verbal se reproduce en los textos no nativos.

Respecto a la interacción entre aspecto léxico y nivel de competencia, nuestro análisis muestra diferencias significativas en los verbos de actividad para el grupo B2 en comparación con C1 y HN. En los textos de B2 se contabilizaron más verbos de actividad en IMP, mientras que en los niveles superiores se impone el IND, construyéndose predicados de coacción aspectual. Se confirma así solo parcialmente nuestra segunda hipótesis, únicamente para los verbos de actividad.

Por otro lado, se constata que los hablantes no nativos muestran una clara tendencia a actualizar los estados en IMP, en la línea de los resultados de Cadierno (2000), y en contra de los Amenós (2010), en cuyas producciones escritas identifica desde niveles tempranos apariciones en IMP e IND con todas las clases de aspecto léxico. En verbos de estado en IND, es decir, predicados no prototípicos, los hablantes de nivel B2 muestran el porcentaje de producción más bajo. La preferencia por el IMP persiste de manera notable en los tres grupos de hablantes no nativos.

Desde la instrucción formal en L2, entendemos que se debería facilitar una exposición a un input que presente formas verbales que contengan combinaciones no prototípicas de aspecto léxico y gramatical que, a pesar de ser menos frecuentes, deben estar disponibles para ser correctamente interpretadas por los hablantes no nativos. En este sentido, propuestas como la de Palacio (2016), centrada en el trabajo de interpretación de verbos télicos (logros) con IMP, marcan un tipo de intervenciones pedagógicas, cuyo objetivo es entrenar a los estudiantes en las reinterpretaciones que emergen de las combinaciones no prototípicas.

\section{CONCLUSIONES}

Los resultados del presente estudio permiten identificar un patrón de desarrollo de la adquisición de las formas verbales de IMP e IND que coincide con la actuación de los hablantes nativos. El grupo de B2 es el único que muestra diferencias significativas en comparación con el nativo. Las composiciones de los tres grupos no nativos son bastante homogéneas, lo que demuestra, tal y como señalan Birdsong y Paik (2008), que el progreso en niveles avanzados de interlengua es más lento que en niveles iniciales. Asimismo, el alto grado de coincidencia con las narraciones del grupo HN pone de manifiesto que es posible alcanzar un nivel de competencia equivalente al nativo en el uso de las formas de pasado para la construcción de un relato (Salaberry, 2018). No obstante, asumimos que para poder confirmar una competencia equivalente a la del hablante nativo son necesarias evidencias empíricas sobre las representaciones que estos hablantes tienen de los valores tempo-aspectuales de estos dos tiempos verbales (Bardovi-Harlig \& Comajoan, 2020), pues, tal y como señala Slabakova (2013, 
2018), es probablemente la interpretación de la morfología funcional, y no el uso, la tarea más compleja y difícil de dominar para el hablante no nativo.

Además de aumentar el número de participantes por grupos, creemos que los hallazgos obtenidos podrían enriquecerse con datos de producción oral y con una prueba con tiempo de realización limitado. Asimismo, teniendo en cuenta que las formas de IMP e IND desempeñan funciones comunicativas diversas, sería necesario ampliar la investigación a usos menos frecuentes de estas formas verbales. En el grupo HN hemos constatado usos del imperfecto narrativo, no registrados en ningún otro grupo. La realización de estudios orientados a analizar fenómenos como este permitiría dilucidar hasta qué punto es válida la constatación del nivel equivalente al nativo que presentan los aprendices de nuestro estudio, especialmente los grupos más avanzados.

\section{REFERENCIAS BIBLIOGRÁFICAS}

Amenós, J. (2010). Los tiempos de pasado del español y el francés: Semántica, pragmática y aprendizaje de E/LE. Perspectivas desde la Teoría de la Relevancia. Tesis doctoral, Universidad Nacional de Educación a Distancia, Madrid, España.

Andersen, R. W. \& Shirai, Y. (1994). Discourse motivations for some cognitive acquisition principles. Studies in Second Language Acquisition, 16, 135-156.

Baralo, M. (2007). La alternancia imperfecto-indefinido en el español no nativo. En P. Cano (Coord.), Actas del VI Congreso de Lingüistica General, Vol. 1 (pp. 339-346). Madrid: Arco Libros.

Bardovi-Harlig, K. (1992). The telling a tale: Discourse structure and tense use in learners' narratives. Pragmatics and Language Learning, 3, 144-161.

Bardovi-Harlig, K. (1995). A narrative perspective on the development of tense/aspect system in second language acquisition. Studies in Second Language Acquisition, 17, 263-291.

Bardovi-Harlig, K. (2000). Tense and aspect in second language acquisition: Form, meaning, and use. Oxford: Blackwell.

Bardovi-Harlig, K. \& Comajoan, Ll. (2020). The aspect hypothesis and the acquisition of L2 past morphology in the last 20 years: A state-of-the-scholarship review. Studies in Second Language Acquisition, 1-31. DOI:10.1017/S0272263120000194

Birdsong, D. \& Paik, J. (2008). Second language acquisition and ultimate attainment. En B. Spolsky \& F. M. Hult (Eds.), The Handbook of Educational Linguistics (pp. 424-436). Oxford: Blackwell. 
Borg, N., O’Hara, J. \& Hutter, C. (2008). Edmodo (Web version) [software] [en línea]. Disponible en: https://www.edmodo.com/

Cadierno, T. (2000). The acquisition of Spanish preterite and imperfect by Danish advanced language learners. Denmark. University of Southern Denmark. Spanish Applied Linguistics, 4, 1-53.

Comajoan, Ll. \& Pérez Saldanya, M. (2005). Grammaticalization and language acquisition: Interaction of lexical aspect and discourse. En D. Eddington (Ed.), Selected Proceedings of the 6th Conference on the Acquisition of Spanish and Portuguese as First and Second Languages (pp. 44-55). Somerville, MA: Cascadilla Press.

Comrie, B. (1976). Aspect: An introduction to verbal aspect and related problems. Cambridge: University Press.

Díaz, L., Bel, A., Ruggia, A., Bekiou, K. \& Rosado, E. (2003). Morphosyntactic interfaces in Spanish L2 acquisition: The case of aspectual differences between indefinido and imperfecto. En J. M. Liceras, H. Goodluck \& H. Zobl (Eds.), Proceedings of the 6th Generative Approaches to Second Language Acquisition Conference: L2 Links (pp. 76-84). Somerville: Cascadilla Press.

Ellis, N. C. (2013). Second language acquisition. En G. Trousdale \& T. Hoffmann (Eds.), Oxford Handbook of Construction Grammar (pp. 365-378). Oxford: Oxford University Press.

Güell, L. (1998). La adquisición del tiempo verbal en el aprendizaje del español como lengua extranjera. Tesis doctoral, Universitat Autònoma de Barcelona, Barcelona, España.

Hasbún, L. (2000). La hipótesis de la distribución sesgada como explicación a comportamientos lingüísticos de los aprendices del español como lengua extranjera. Filología y Lingüistica, 26(2), 235-246.

Housen, A. (1994). Tense and aspect in second language acquisition: The Dutch interlanguage of a native speaker of English. En C. Vet \& C. Vetters (Eds.), Tense and Aspect in Discourse (pp. 257-292). Berlin: Mouton de Gruyter.

Howard, M. \& Leclercq, P. (Eds.) (2017). Tense-aspect-modality in a second language: Comtemporary perspectives. Ámsterdam: John Benjamins.

Labeau, E. (2005). Beyond the aspect hypothesis. Tense-aspect development in advanced L2 French. EUROSLA Yearbook, 5, 77-101.

Labeau, E. \& Saddour, I. (Eds.) (2012). Tense, aspect and mood in first and second language acquisition. Amsterdam: Rodopi. 
Li, P. \& Shirai, Y. (2000). The acquisition of lexical and grammatical aspect. Berlin: Mouton de Gruyter.

Liskin-Gasparro, J. (1996). Narrative strategies: A case study of developing storytelling skills by a learner of Spanish. The Modern Language Journal, 80, 271-286.

Liskin-Gasparro, J. (2000). The use of tense-aspect morphology in Spanish oral narratives: Exploring the perceptions of advanced learners. Hispania, 83(4), 830-844.

Llopis-García, R., Real Espinosa, J. \& Ruiz Campillo, J. (2012). Qué gramática enseñar, qué gramática aprender. Madrid: Edinumen.

López Ortega, N. (2000). Tense, aspect, and narrative structure in Spanish as a second language. Hispania, 83(3), 488-502.

MacWhinney, B. (2000). The CHILDES project: tools for analyzing talk. Mahwah, NJ: Lawrence Erlbaum.

Mañas, I. (2011). Dos maneras de viajar al pasado. Estudio comparativo de la expresión del pasado en ruso y español. Cuadernos de Rusística Española, 7, 2940.

Montrul, S. \& Salaberry, R. (2003). The development of tense/aspect morphology in Spanish as a second language. En B. A. Lafford \& R. Salaberry (Eds.), Spanish second language acquisition (pp. 47-73). Washington, DC: Georgetown University Press.

Montrul, S. \& Slabakova, R. (2003). Competence similarities between native and nearnative speakers: An investigation of the Preterite-Imperfect contrast in Spanish. Studies in Second Language Acquisition, 25, 351-398.

Ortiz Gozalo, J. M. (1994). Valor discursivo de la oposición imperfecto/indefinido en el relato. Revista de Filología Románica, 11-12, 187-216.

Palacio, B. (2016). Gramática cognitiva-operativa: limitaciones de una instrucción única. El caso de imperfecto/indefinido en el aula de ELE. MarcoELE, 22 [en línea] Disponible en: http://marcoele.com/descargas $/ 22 /$ palacioimperfecto indefinido.pdf

Polio, C. (2012). How to research language writing. En A. Mackey \& S. Gass (Eds.), Research Methods in Second Language Acquisition: A Practical Guide (pp. 139-157). Oxford: Blackwell.

Reinhart, T. (1984). Principles of Gestalt perception in the temporal organization of narrative texts. Linguistics, 22, 779-809. 
Salaberry, R. M. (1999). The development of past tense verbal morphology in classroom L2 Spanish. Applied Linguistics, 20, 151-178.

Salaberry, R. M. (2011). Assessing the effect of Lexical Aspect and Grounding on the Acquisition of L2 Spanish Paste Tense Morphology. Bilingualism: Language and Cognition, 4(2), 184-202.

Salaberry, R. M. (2013). Contrasting Preterite and Imperfect use among advanced L2 learners: Judgements of iterated eventualities in Spanish. IRAL, 51, 243-270.

Salaberry, R. M. (2018). Advanced Conceptualizations of Tense and Aspect in L2 Acquisition. En P. A. Malovrh \& A. G.Benati (Eds.), The Handbook of Advanced Proficiency in Second Language Acquisition (pp. 361-380). Oxford: John Wiley.

Salaberry, R. M. \& Comajoan, Ll. (Eds.) (2013). Research desgin and methodology in studies on L2 tense and aspect. Boston/Berlín: De Gruyter Mouton.

Salaberry, R. M. \& Shirai, Y. (Eds.) (2002). Tense-aspect morphology in L2 acquisition. Amsterdam/Philadelphia: John Benjamins.

Schell, K. (2000). Functional categories and the acquisition of aspect in L2 Spanish: A longitudinal study. Tesis doctoral, University of Washignton, Seattle, USA.

Shirai, Y. \& Andersen, W. (1995). The acquisition of tense-aspect morphology: A prototype account. Language, 71(4), 743-762.

Slabakova, R. (2013). What is easy and what is hard to acquire in a second language. En M. P. García Mayo, M. J. Gutiérrez \& M. Martínez (Eds.), Contemporary Approaches to Second Language Acquisition (pp. 5-28). Amsterdam: John Benjamins.

Slabakova, R. (2018). Inflectional Morphology. En P. A. Malovrh \& A. G. Benati (Eds.), The Handbook of Advanced Proficiency in Second Language Acquisition (pp. 381-400). Oxford: John Wiley.

Slabakova, R. \& Montrul, S. (2008). Aspectual shifts: Grammatical and pragmatic knowledge in L2 acquisition. En J. M. Liceras, H. Zobl \& H. Goodluck (Eds.), The Role of Formal Features in Second Language Acquisition (pp. 452-483). Nueva York: Lawrence Erlbaum Associates.

Smith, C. (1991). The parameter of aspect. Dordrecht: Kluwer Academic Publishers.

Swart, H. (1998). Aspect shift and coercion. Natural Language and Linguistic Theory, 16, 347-385. 